\title{
On the Specific On-state Resistance of Superjunction MOSFETs with a Compensated Pillar
}

\author{
H. Kang and F. Udrea, Member, IEEE
}

\begin{abstract}
In this paper, we report an analytical model for the on-state characteristics of a superjunction MOSFET featuring a compensated pillar between the n-pillar and the p-pillar is established. Since a large amount of lateral electric field is sustained in the compensated region, the doping concentration in the n-pillar can be enhanced significantly, leading to a substantial reduction in the on-state resistance of the superjunction. Simulation results proved that the use of an extra compensated pillar within the superjunction structure reduces the on-state resistance by $25 \%$ compared to that of a conventional superjunction for the same breakdown voltage.
\end{abstract}

Index Terms-Superjunction, JFET, Compensated.

\section{INTRODUCTION}

The reduction in specific resistance, $R_{s p}$, of superjunction (SJ) metal-oxide-semiconductor field effect transistors (MOSFETs) is a key factor in meeting application requirements, such as high energy efficiency and reducing the fabrication cost [1]. For this, several novel ideas have been suggested, such as the use of an oxide field plate between the n-pillar and p-pillar (p/oxid/n) [2], p/n/high-k dielectric pillar [3], a gate-driven accumulated pillar [4], p/n/oxide/undoped poly-Si/oxide pillar [5] and three-dimensional hexagonal pillar [6]. However, the above structures require additional fabrication steps (increased cost) and/or are subject to reliability challenges such as hot carrier injection (HCI) and time dependent dielectric breakdown (TDDB).

Here we discuss an alternative structure to reduce the $R_{s p}$ in a superjunction MOSFET while maintaining a conventional fabrication process. As shown in Fig. 1, a compensated pillar is inserted in between the p-type and the n-type pillar. The compensated pillar can be formed by deep diffusion or overlapping implantations to yield the same amount of the n-type dopant and the p-type dopant in a certain width, $\gamma d(0<\gamma<1)$. Even though the compensated (intrinsic) region decreases the conducting path of the n-pillar, the parasitic depletion width toward the n-pillar can be reduced by increasing the doping concentration. Also, a large amount of the drain to source voltage, $V_{D S}$, is to be sustained across the compensated region as shown in Fig. 1 and, therefore, the parasitic depletion width in the n-pillar can be reduced more than that of the conventional superjunction structure $(\gamma=0)$. This study provides an analytic model for a superjunction MOSFET with a compensated pillar and investigates the advantages over the conventional superjunction MOSFETs. For this, we do not take into account the channel resistance

H. Kang is with the Electrical Engineering Department of University of Cambridge, 9 JJ Thomson Avenue, CB30FA, Cambridge, U.K. (email: hk428@cam.ac.uk)

F. Udrea (Corresponding author) is with the Electrical Engineering Department of University of Cambridge, 9 JJ Thomson Avenue, CB30FA, Cambridge, U.K. (email: fu@eng.cam.ac.uk); and we assume that the doping concentrations $\left(N_{D}\right.$ and $\left.N_{A}\right)$ and the widths of the p-pillar and the n-pillar are the same for the charge balance.

(a)

(b)

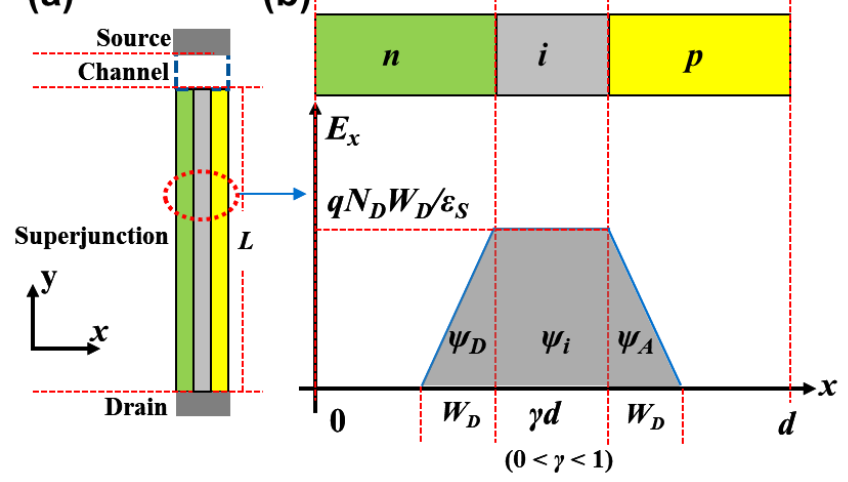

Fig. 1. A schematic illustration of (a) a compensated pillar superjunction structure and (b) the lateral electric field and the depletion width in a compensated superjunction at a given potential.

\section{IDEAL APPROACH}

The width of the compensated pillar is $\gamma d(0<\gamma<1)$ and the compensated pillar is located in the centre of the superjunction structure as shown in Fig. 1. The depletion width towards the conducting n-pillar, $W_{D}$, was ignored The ideal $R_{s p . i d e a l}$ of this structure can be written as

$$
R_{\text {sp.ideal }}=\frac{1}{q N_{D} \mu_{n}} \frac{L}{Z(d-\gamma d) / 2} d Z=\frac{2 L}{q N_{D} \mu_{n}} \frac{1}{(1-\gamma)}
$$

where $q, \mu_{n}, Z$, and $L$ are unit charge, doping dependent electron mobility, depth of the pillar (the $3^{\text {rd }}$ dimension into the paper), and the length of the pillar, respectively. When the superjunction is fully depleted, the lateral electric field, $E_{x}$ should be less than the critical electric field, $E_{C}[7]$ :

$E_{x}=\alpha E_{C}=\frac{q N_{D}}{\varepsilon_{S}}\left(\frac{d-\gamma d}{2}\right),(0<\alpha<1)$.

where $\varepsilon_{S}$ is the permittivity of the semiconductor material. Since the square of the critical electric field, $E_{C}{ }^{2}$, is the sum of the square of the lateral electric field, $E_{x}^{2}$, and the square of the vertical electric field, $E_{y}{ }^{2}$, the vertical electric field becomes

$E_{y}=\sqrt{\left(1-\alpha^{2}\right)} E_{C}$.

The breakdown voltage, $V_{B}$, is the sum of the voltage drops across the doped pillar region $V_{L}$, the compensated region $V_{i}$, and the vertical voltage drop $V_{V}$. Assuming the length of the superjunction, $L$, is relatively long compared to the cellpitch, $d$, the breakdown voltage, $V_{B}$ can be written as $V_{B}=V_{L}+V_{i}+V_{V}$

$=\alpha E_{C} \frac{(1+\gamma)}{2} d+E_{C} L \sqrt{1-\alpha^{2}} \approx E_{C} L \sqrt{1-\alpha^{2}}$. 
By inserting equations (2) and (4) into (1), the ideal $R_{s p}$ has the following material form,

$R_{\text {sp.ideal }}=\frac{V_{B}}{\alpha \sqrt{1-\alpha^{2}} \mu_{n} \varepsilon_{S} E_{C}{ }^{2}} d=\frac{2 V_{B}}{\mu_{n} \varepsilon_{S} E_{C}{ }^{2}} d$,

where $\alpha$ is $1 / \sqrt{2}$ to minimize the $R_{s p . i d e a l}$. The material figure of merit (FOM) $V_{B} / R_{s p}$ given by equation (5) is the same as the previously reported ideal FOM of a conventional superjunction MOSFET $(\gamma=0)$ [7]-[9].

\section{JFET APPROACH}

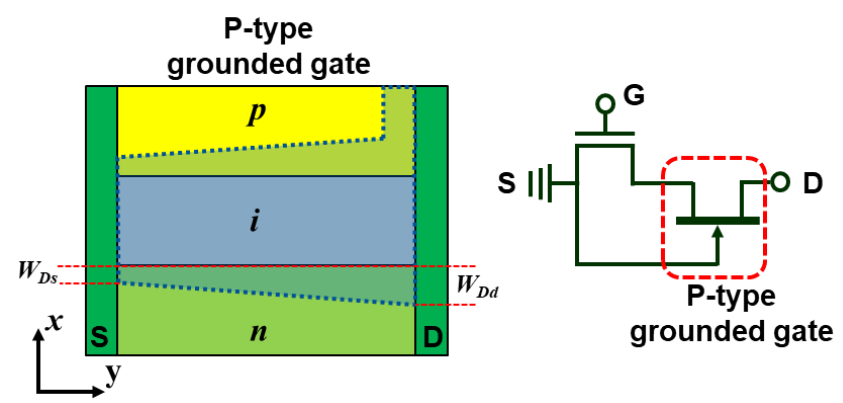

Fig. 2. A schematic illustration of depletion width profiles in a compensated pillar superjunction and its JFET inner circuit.

For a $V_{D S}$ dependent analysis of a compensated pillar superjunction $R_{s p}$, a grounded gate junction field effect transistor (JFET) model is provided in Fig. 2 [8], [9]. The p-pillar of the superjunction is the gate of the JFET (grounded) and the n-pillar is the channel of the JFET. The depletion width, $W_{D s}$, is formed by the built-in potential, $\psi_{b i}$, and $W_{D d}$ is formed by $\psi_{b i}+V_{D S}$. As shown in Fig. 1 and 2, the potential, $\psi(y)$, induced by $\psi_{b i}+V_{D S}$ is sustained across the $\mathrm{p}$-i-n region and, the depletion width (before pinch-off of the pillars), $W_{D}$, has the following relationship:

$\psi(y)=\psi_{D}+\psi_{A}+\psi_{i}=\frac{q N_{D}}{\varepsilon_{S}} W_{D}^{2}+\frac{q N_{D}}{\varepsilon_{S}} W_{D} \gamma d$.

Where the $\psi_{D}$ and $\psi_{A}$ are the same due to the symmetrical structure. From equation (6), $W_{D s}$ and $W_{D d}$, become

$$
\begin{aligned}
& \sqrt{\frac{\varepsilon_{S} \psi_{b i}}{q N_{D}}+\frac{\gamma^{2} d^{2}}{4}-\frac{\gamma d}{2}}=W_{D s}, \\
& \sqrt{\frac{\varepsilon_{S}\left(\psi_{b i}+V_{D S}\right)}{q N_{D}}+\frac{\gamma^{2} d^{2}}{4}}-\frac{\gamma d}{2}=W_{D d} .
\end{aligned}
$$

Based on the above depletion widths, the sheet charge density, $Q_{n}\left(\mathrm{~cm}^{-2}\right)$, in the n-pilar leads to

$Q_{n}(y)=q N_{D}\left(\frac{d-\gamma d}{2}-W_{D}(y)\right)$.

The drain current, $I_{D}$, has the following relationship

$I_{D}=\frac{Z}{L} \int_{0}^{L} Q_{n}(y) v(y) d y$,

where $v(y)$ is the electron velocity in the n-pillar:

$v(y)=\mu_{n} \frac{\partial \psi}{\partial y}$.

By inserting equations (6), (7), (8) and (10) into (9), and solving equation (9), the drain current leads to

$$
\begin{aligned}
& I_{D}=\frac{Z \mu_{n} q^{2} N_{D}^{2}}{\varepsilon_{S} L} \times \\
& \left\{-\frac{2}{3}\left[\left(W_{D d}+\frac{\gamma d}{2}\right)^{3}-\left(W_{D s}+\frac{\gamma d}{2}\right)^{3}\right]+\frac{d}{2} \frac{\varepsilon_{S} V_{D S}}{q N_{D}}\right\}
\end{aligned}
$$

From equation (11), the $R_{s p}$ with respect to the applied $V_{D S}$ for a compensated superjunction can be obtained as

$$
\begin{aligned}
& R_{s p}=\frac{V_{D S}}{I_{D}} d Z=\frac{\varepsilon_{S} d L}{\mu_{n} q^{2} N_{D}{ }^{2}} \times \\
& -\frac{2}{3}\left[\left(W_{D d}+\frac{\gamma d}{2}\right)^{3}-\left(W_{D S}+\frac{\gamma d}{2}\right)^{3}\right]+\frac{d}{2} \frac{\varepsilon_{S} V_{D S}}{q N_{D}}
\end{aligned}
$$

When the $V_{D S}$ approach zero, the $R_{s p}$ given by equation (12) has a minimum value:

$$
\begin{aligned}
& \lim _{V_{D S} \rightarrow 0} R_{s p}\left(V_{D S}\right)=\frac{2 L}{\mu_{n} q N_{D}} \frac{1}{1-\gamma} \frac{d-\gamma d}{d-\gamma d-2 W_{D s}} \\
& =R_{\text {sp.ideal }} \times \frac{d-\gamma d}{d-\gamma d-2 W_{D s}}
\end{aligned}
$$

Equation (13) is the multiplication form of $R_{\text {sp.ideal }}$ by the conducting path ratio where the conducting path is narrowed by the built-in depletion width, $W_{D s}$. If the width of the compensated pillar becomes zero, equation (13) leads to the conventional superjunction $R_{s p}$ [8], [9]:

$$
\lim _{\gamma \rightarrow 0} \frac{2 L}{\mu_{n} q N_{D}} \frac{1}{1-\gamma} \frac{d-\gamma d}{d-\gamma d-2 W_{D s}}=\frac{2 L}{\mu_{n} q N_{D}} \frac{d}{d-2 W_{D s}} .
$$

Therefore, the $R_{s p}$ forms given by equations (12) and (13) are compatible with the conventional superjunction (without a compensated pillar) MOSFETs.

Fig. 3 shows the $R_{s p}$ ratio of the compensated SJ $(0<\gamma$ $<1)$ and the conventional SJ $(\gamma=0)$. The concentration of the pillars at a given cellpitch can be calculated from equation (2):

$$
\frac{1}{2} N_{D}=\frac{1}{2}\left(\frac{1}{\sqrt{2}} \frac{E_{C} \varepsilon_{S}}{q}\left(\frac{2}{d-\gamma d}\right)\right) \text {. }
$$

For a practical approach, the concentrations of the pillars were the half of the optimum value. The critical electric field models were borrowed from Baliga's study [10]:

$$
E_{C}(S i)=4010 \times\left(N_{D}\right)^{1 / 8} \text {, }
$$

The electric field dependent mobility at a given $V_{D S}$ follows a previously established empirical model [8], [9],

$$
\mu_{n}\left(V_{D S}\right)=\mu_{n 0} /\left(1+\left(\frac{V_{D S}}{E_{C} L}\right)^{\frac{1}{m}}\right)^{3 m}, m=1.32+4 \gamma .
$$

Where $\mu_{n 0}$ is the mobility when $V_{D S}$ is $0 \mathrm{~V}$.

As shown in Fig. 3, the Si SJ with a compensated pillar shows a reduction in the $R_{s p}$ by $10 \sim 30 \%$ compared to the conventional $\mathrm{SJ}$ and the reduction is further enhanced as $V_{D S}$ increases up to $3 \mathrm{~V}$. This is due to the fact that the parasitic JFET effect, which obstructs the current conduction path, is less significant in the compensated pillar SJ than in the conventional SJ. The reason for this can be easily understood from Fig. 1 where a large portion of the potential is dropped across the compensated pillar region, rather than in the n-pillar. 
Fig. 4 shows the drain current simulation with respect to the applied $V_{D S}$. The analytical model given by equation (11) showed a good agreement with the simulation result and the compensated pillar structure showed $R_{s p}$ reduction of $\sim 25 \%$ over the conventional structure owing to the high n-pillar doping concentration. Fig. 5 shows the breakdown voltage simulation. The compensated superjunction $V_{B}$ was very similar to that of the conventional superjunction and this should be attributed to the flat lateral electric profile in the compensated pillar. As can be seen in Fig. 5(a), the electric field profile in the compensated region is flat with a high value and, therefore, it can sustain more drain voltage even with a high doping concentration.

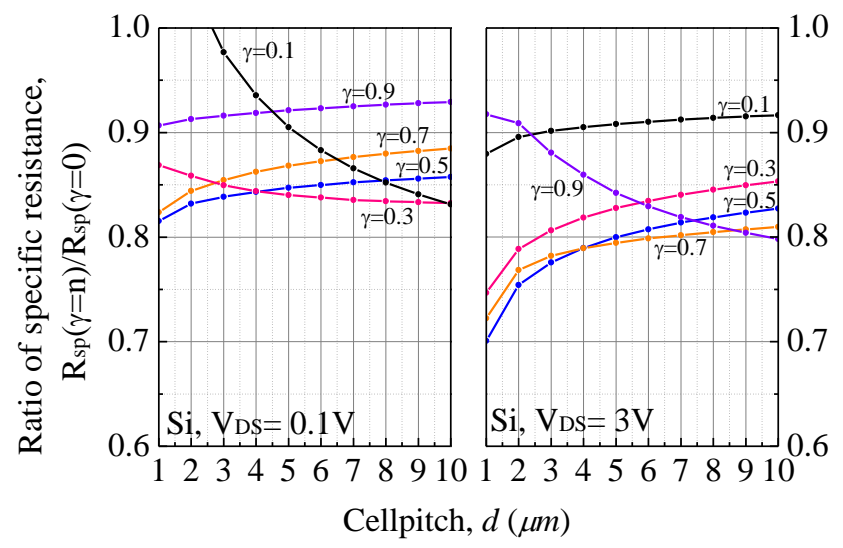

Fig. 3. $R_{s p}$ ratio of the compensated SJ $(0<\gamma<1)$ and the conventional SJ $(\gamma=0)$ with respect to the cellpitch.

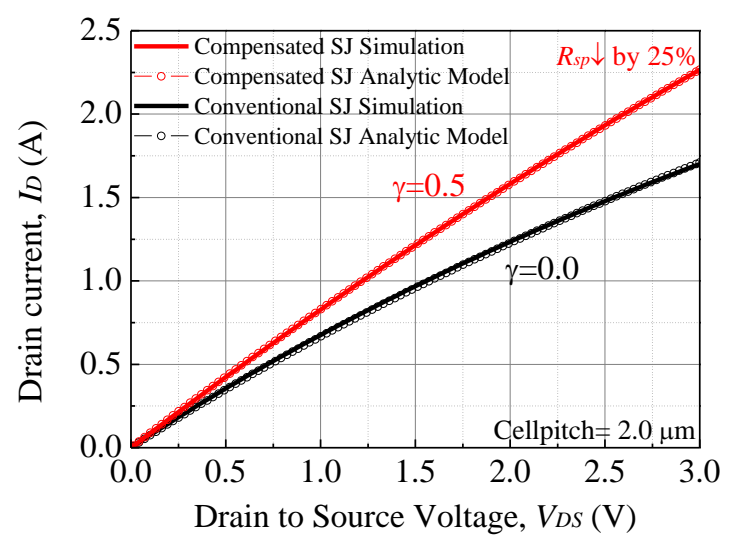

Fig. 4. TCAD drain current simulation result and analytic model in this study for the compensated pillar (red) and the conventional pillar (black) when the cellpitch is $d=2 \mu \mathrm{m}$. The length of pillar, $L=40 \mu \mathrm{m}$, the number of cells: $2.0 \times 10^{5}, N_{D}(\gamma=0.5)=2.19 \times 10^{16} \mathrm{~cm}^{-3}$, and $N_{D}(\gamma=0)=9.91$ $\times 10^{15} \mathrm{~cm}^{-3}$.

Fig. 6 presents inductive switching characteristics for the devices given by Figs. 4 and 5. The parasitic inductances were ignored. Both devices show similar $d v / d t$ and $d i / d t$ during turn-on and turn-off. The only difference, though not significant, is the rapid drain voltage rising point (conventional pillar: $14 \mathrm{~V}$ and compensated pillar: $26 \mathrm{~V}$ ) during the turn-off where the accumulation region below the gate oxide becomes depleted. As previously reported [11], a highly doped n-pillar below the gate oxide requires a higher drain voltage to be depleted.
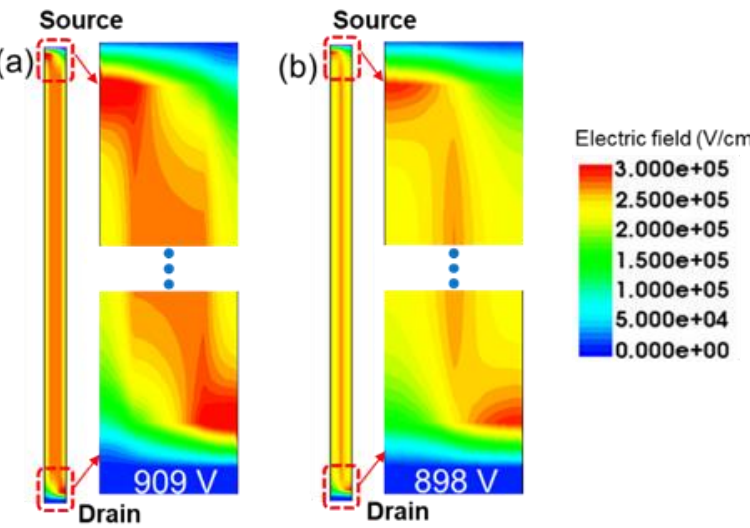

Fig. 5. $2 \mathrm{D}$ TCAD breakdown voltage simulation result and the electric field profile for (a) the compensated pillar (b) the conventional pillar when the cellpitch is $d=2 \mu \mathrm{m}$. The length of pillar, $L=40 \mu \mathrm{m}, N_{D}(\gamma=0.5)=$ $2.19 \times 10^{16} \mathrm{~cm}^{-3}$, and $N_{D}(\gamma=0.5)=9.91 \times 10^{15} \mathrm{~cm}^{-3}$.

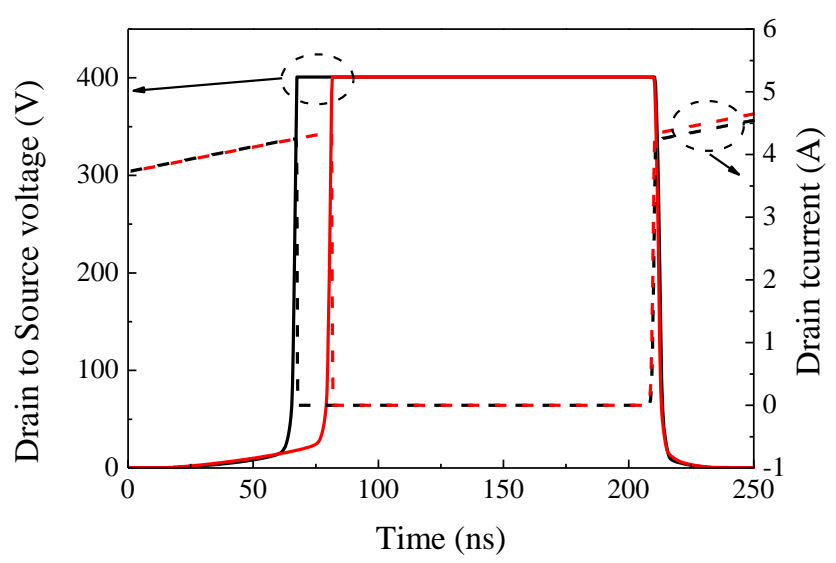

Fig. 6. TCAD inductive switching simulation result for the conventional SJ (black, $\gamma=0$ ) and the compensated pillar (red, $\gamma=0.5$ ) when the cellpitch is $d=2 \mu \mathrm{m}$. The length of pillar, $L=40 \mu \mathrm{m}$, the number of cells: $4.0 \times 10^{6}, N_{D}(\gamma=0)=9.91 \times 10^{15} \mathrm{~cm}^{-3}$ and $N_{D}(\gamma=0.5)=2.19 \times 10^{16} \mathrm{~cm}^{-3}$.

\section{Conclusion}

An analytical model for a compensated pillar SJ MOSFET has been developed by using a grounded JFET theory. This model accurately predicts the on-state resistance and the drain current at a given drain voltage. Also, this model is compatible with the conventional SJ MOSFET. It has been found that the compensated pillar SJ MOSFET can reduce the $R_{s p}$ by $10 \sim 30 \%$ compared to that of conventional SJ MOSFET.

\section{ACKNOWLEDGEMENT}

This research is supported by On Semiconductor Corporation. The authors are particularly grateful to Thomas Neyer for his valuable advice on this study and his general support of this research.

\section{REFERENCES}

[1] F. Udrea, G. Deboy, S. Member and T. Fujihira, "Superjunction Power Devices, History, Development, and Future Prospects," IEEE Trans. Electron Devices, vol. 64, no. 3, pp. 713-727, Jan. 2017. 
[2] W. Saito, "Comparison of Theoretical Limits between Superjunction and Field Plate Structures," Proc. Int. Symp. Power Semicond. Devices ICs, pp. 241-244, May 2013.

[3] X. Luo, Y. H. Jiang, K. Zhou, P. Wang, X. W. Wang, Q. Wang, G. L. Yao, B. Zhang and Z. J. Li, "Ultralow Specific On-Resistance Superjunction Vertical DMOS With High-k Dielectric Pillar," IEEE Electron Device Lett., vol. 33, no. 7, pp. 1042-1044, Jun. 2012.

[4] W. Saito, "Breakthrough of drain current capability and on-resistance limits by gate-connected superjunction MOSFET," 2018 IEEE 30th Int. Symp. Power Semicond. Devices ICs, pp. 36-39, May 2018.

[5] Z. Cao, B. Duan, T. Shi, S. Yuan and Y. Yang, "A Superjunction U-MOSFET With SIPOS Pillar Breaking Superjunction Silicon Limit by TCAD Simulation Study," IEEE Electron Device Lett., vol. 38, no. 6, pp. 794-797, Apr. 2017.

[6] J. Park and J. H. Lee, “A 650 V Super-Junction MOSFET With Novel Hexagonal Structure for Superior Static Performance and High BV Resilience to Charge Imbalance: A TCAD Simulation Study," IEEE Electron Device Lett., vol. 38, no. 1, pp. 111-114, Nov. 2017.

[7] T. Fujihira, "Theory of semiconductor superjunction devices," Japanese J. Appl. Physics, Part 1 Regul. Pap. Short Notes Rev. Pap., vol. 36, no. 10, pp. 6254-6262, Oct. 1997.

[8] H. Kang and F. Udrea, "True Material Limit of Power Devices -Applied to 2-D Superjunction MOSFET," IEEE Trans. Electron Devices, vol. 65, no. 4, pp. 1432-1439, Mar. 2018.

[9] H. Kang and F. Udrea, "Material Limit of Power Devices--Applied to Asymmetric 2-D Superjunction MOSFET," IEEE Trans. Electron Devices, pp. 1-7, May 2018.

[10] B. J. Baliga, Fundamentals of Power Semiconductor Devices. Springer Science + Business Media, LLC, 2010.

[11] Y. Xiong, S. Sun, H. Jia, P. Shea and Z. J. Shen, "New Physical Insights on Power MOSFET Switching Losses," IEEE Trans. Power Electron., vol. 24, no. 2, pp. 525-531, Feb. 2009. 